The Astrophysical Journal, 674:1068-1077, 2008 February 20

(C) 2008. The American Astronomical Society. All rights reserved. Printed in U.S.A.

\title{
FLUORINE IN R CORONAE BOREALIS STARS
}

\author{
GaJendRa PANDEY \\ Indian Institute of Astrophysics; Bangalore, 560034 India; pandey@iiap.res.in \\ DAVID L. LAMBerT \\ The W.J. McDonald Observatory, University of Texas, Austin, TX 78712-1083; dll@astro.as.utexas.edu \\ AND \\ N. Kameswara RaO \\ Indian Institute of Astrophysics, Bangalore, 560034 India; nkrao@iiap.res.in \\ Received 2007 October 11; accepted 2007 November 14
}

\begin{abstract}
Neutral fluorine ( $\mathrm{F}$ I) lines are identified in the optical spectra of several R Coronae Borealis stars (RCBs) at maximum light. These lines provide the first measurement of the fluorine abundance in these stars. Fluorine is enriched in some RCBs by factors of $800-8000$ relative to its likely initial abundance. The overabundances of fluorine are evidence for the synthesis of fluorine. These results are discussed in the light of the scenario that RCBs are formed by accretion of an He white dwarf by a $\mathrm{C}-\mathrm{O}$ white dwarf. Sakurai's object (V4334 Sgr), a final He-shell flash product, shows no detectable F I lines.
\end{abstract}

Subject headings: stars: abundances — stars: chemically peculiar — stars: evolution

\section{INTRODUCTION}

$\mathrm{R}$ Coronae Borealis ( $\mathrm{RCB}$ ) stars comprise a sequence of hydrogen-deficient supergiants with effective temperatures from about $T_{\text {eff }}=3500 \mathrm{~K}$, as represented by Z UMi and DY Per, to about $19,500 \mathrm{~K}$, as represented by DY Cen. The characteristic of $\mathrm{H}$ deficiency is shared by the $\mathrm{H}$-deficient cool carbon ( $\mathrm{HdC}$ ) stars at low temperatures and by the extreme helium (EHe) stars at high temperatures. A common assumption is that the sequence HdC-RCB-EHe in the $\left(T_{\text {eff }}, \log g\right)$ plane reflects a close evolutionary connection. In this sequence, the RCBs are distinguished from $\mathrm{HdC}$ and $\mathrm{EHe}$ stars by their second principal defining characteristic: their propensity to fade, in visual light, unpredictably as a cloud of carbon dust obscures the star. This propensity is not universally shared: XX Cam has yet to be observed below maximum light. In addition, DY Cen might be considered as an $\mathrm{EHe}$ star known to experience RCB-like fadings.

If $\mathrm{HdC}, \mathrm{RCB}$, and $\mathrm{EHe}$ stars share a common heritage, the expectation is that their atmospheric compositions should show some common features (Pandey et al. 2004b; Rao 2005). It is through the compositions that one hopes to test theoretical ideas about the origins of these extremely rare stars; just five $\mathrm{HdC}$, about 40 RCB (Zaniewski et al. 2005), and 21 EHe stars are known in the Galaxy. Currently, two scenarios remain in contention to account for these H-poor high-luminosity stars. In the first, a final He-shell flash in a post-AGB star on the white dwarf cooling track creates a H-poor luminous star. This is dubbed the "final flash" (FF) scenario. In the second, the H-poor star is formed from a merger of a $\mathrm{He}$ white dwarf with a $\mathrm{C}-\mathrm{O}$ white dwarf. In a close binary system, accretion of the He white dwarf by the $\mathrm{C}-\mathrm{O}$ white dwarf may lead to a H-poor supergiant with the $\mathrm{C}-\mathrm{O}$ white dwarf as its core. This is called the "double degenerate" (DD) scenario.

Products of both the FF and DD scenarios may be presumed to exist. A determination of which scenario provided which star rests in large part on the observed chemical composition of a star's atmosphere and theoretical predictions about the FF and DD products. Evidence from elemental abundances, especially the
$\mathrm{H}, \mathrm{C}, \mathrm{N}$, and $\mathrm{O}$ abundances, suggests that the RCB and EHe stars evolved via the DD rather than the FF route (Asplund et al. 2000; Pandey et al. 2001, 2006; Saio \& Jeffery 2002). Convincing, essentially incontrovertible, evidence that the DD scenario led to the HdCs and some cool RCBs was presented by Clayton et al. (2007) with their discovery that the ${ }^{18} \mathrm{O}$ was very abundant in their atmospheres. This usually rare isotope of oxygen was attributed to nucleosynthesis occurring during and following accretion of the He-rich material onto the $\mathrm{C}-\mathrm{O}$ white dwarf.

Determination of the oxygen isotopic ratios demands a cool star with the $\mathrm{CO}$ vibration-rotation bands in its spectrum. The majority of RCBs and all of the EHes are too hot for CO to contribute to their spectra (Tenenbaum et al. 2005). ${ }^{1}$ An alternative tracer of nucleosynthesis during a merger may be provided by the fluorine abundance. Considerable enrichment of EHe stars with F was discovered by Pandey (2006) from detection and analysis of about a dozen F I lines in optical spectra of cool EHe stars. Clayton et al.'s (2007) calculations suggest that F synthesis is possible in the DD scenario. In this paper, we report on a search for F $\mathrm{I}$ lines in spectra of RCBs and discuss the F abundances in light of the results for EHes and the expectations for the DD and FF scenarios.

\section{OBSERVATIONS}

High-resolution optical spectra of RCBs at maximum light obtained at the W. J. McDonald Observatory and the Vainu Bappu Observatory were examined for the presence of $F_{\text {I lines. A key }}$ requirement of the spectra for inclusion in the study was that the absorption line profiles be symmetric; an asymmetry and a doubling of the lines is attributable to a pulsation of the atmosphere that may induce a shock wave. An additional requirement was that the signal-to-noise ratio be adequate for detection of weak lines.

\footnotetext{
${ }^{1}$ A possibility exists that circumstellar $\mathrm{CO}$ may be detectable for the RCBs. Cool gas containing $\mathrm{C}_{2}$ molecules has been seen for $\mathrm{V} 854 \mathrm{Cen}, \mathrm{R} \mathrm{CrB}$, and $\mathrm{V} \mathrm{CrA}$ when the stars were in decline (Rao \& Lambert 2000, 2007; Rao et al. 2006).
} 
TABLE 1

The Analyzed RCBs, Their Stellar Parameters, and Fluorine Abundances from Individual F i Lines; Sakurai's Object, a Final He-Shell Flash Product, Is Also Listed

\begin{tabular}{|c|c|c|c|c|c|c|c|}
\hline \multirow[b]{2}{*}{$\mathrm{S}_{\mathrm{TAR}}$} & \multirow[b]{2}{*}{$\left(T_{\mathrm{eff}}, \log g, \xi\right)$} & \multicolumn{6}{|c|}{$\log \epsilon(\mathrm{F})(\AA)$} \\
\hline & & 7398.68 & 7754.69 & 6902.47 & 7425.6 & 6834.26 & Mean \\
\hline V3795 Sgr $^{\mathrm{a}} \ldots \ldots \ldots \ldots \ldots$ & $(8000,1.0,10.0)$ & $\ldots$ & 6.60 & 6.65 & 6.70 & 6.70 & $6.66 \pm 0.05(4)$ \\
\hline UW Cen ........................ & $(7500,1.0,12.0)$ & 7.20 & $\ldots$ & 7.00 & 7.10 & 7.20 & $7.1 \pm 0.1(4)$ \\
\hline RY Sgr ........................... & $(7250,0.75,6.0)$ & $\ldots$ & $\ldots$ & 6.80 & $\ldots$ & 7.10 & $6.95 \pm 0.2(2)$ \\
\hline XX Cam.......................... & $(7250,0.75,9.0)$ & $\ldots$ & $\ldots$ & $<5.6$ & $\ldots$ & $<5.6$ & $<5.6$ \\
\hline UV Cas .......................... & $(7250,0.5,7.0)$ & $\ldots$ & $\ldots$ & 6.20 & $\ldots$ & $\ldots$ & $6.2(1)$ \\
\hline UX Ant ........................ & $(7000,0.5,5.0)$ & $\ldots$ & $\ldots$ & $<6.2$ & $\ldots$ & $<6.2$ & $<6.2$ \\
\hline $\mathrm{VZ} \mathrm{Sgr}^{\mathrm{a}} \ldots \ldots \ldots \ldots \ldots \ldots$ & $(7000,0.5,8.0)$ & $\ldots$ & $\ldots$ & 6.30 & $\ldots$ & 6.50 & $6.4 \pm 0.1(2)$ \\
\hline $\mathrm{R} \mathrm{CrB}$ & $(6750,0.5,7.0)$ & $\ldots$ & $\ldots$ & 6.85 & $\ldots$ & 7.00 & $6.9 \pm 0.1(2)$ \\
\hline V2552 Oph .................... & $(6750,0.5,7.0)$ & $\ldots$ & $\ldots$ & 6.60 & $\ldots$ & $\ldots$ & $6.6(1)$ \\
\hline V854 Cen ${ }^{\mathrm{a}} \ldots \ldots \ldots \ldots \ldots . . . . . . . .$. & $(6750,0.0,6.0)$ & $\ldots$ & $\ldots$ & $\ldots$ & $\ldots$ & $<5.7$ & $<5.7$ \\
\hline SU Tau .......................... & $(6500,0.5,7.0)$ & $\ldots$ & $\ldots$ & 6.90 & $\ldots$ & 7.00 & $6.95 \pm 0.1(2)$ \\
\hline $\mathrm{V} \mathrm{CrA}^{\mathrm{a}}$ & $(6500,0.5,7.0)$ & $\ldots$ & $\ldots$ & $6.5:$ & $\ldots$ & $\ldots$ & $6.5:(1)$ \\
\hline V482 Cyg....................... & $(6500,0.5,4.0)$ & $\ldots$ & $\ldots$ & $\ldots$ & $\ldots$ & 6.6: & $6.6:(1)$ \\
\hline GU Sgr........................ & $(6250,0.5,7.0)$ & $\ldots$ & $\ldots$ & $\ldots$ & $\ldots$ & 7.2: & $7.2:(1)$ \\
\hline FH Sct.......................... & $(6250,0.25,6.0)$ & $\ldots$ & $\ldots$ & $\ldots$ & $\ldots$ & $7.2:$ & $7.2:(1)$ \\
\hline Sakurai's object.......... & $(7500,0.0,8.0)$ & $\ldots$ & $\ldots$ & $\ldots$ & $\ldots$ & $<5.4$ & $<5.4$ \\
\hline
\end{tabular}

${ }^{\mathrm{a}}$ Minority RCBs.

Spectra from the McDonald Observatory were obtained with the $2.7 \mathrm{~m}$ Harlan J. Smith Telescope and the coudé cross-dispersed echelle spectrometer ( Tull et al. 1995) at a resolving power $R=$ $\lambda / \Delta \lambda=60,000$ with a bandpass covering much of the $3800-$ $10000 \AA$ interval.

At the Vainu Bappu Observatory (VBO), spectra were acquired with the Vainu Bappu Telescope with a fiber-fed cross-dispersed echelle spectrometer (Rao et al. 2004, 2005). These spectra were acquired with two different grating settings to cover the wavelength regions of the crucial $\mathrm{F}_{\mathrm{I}}$ lines and are at a resolving power $R \simeq 30,000$ with a wavelength coverage of 4860-7900 $\AA$.

All spectra were reduced in standard fashion using the Image Reduction and Analysis Facility (IRAF) software. ${ }^{2}$ The reduction procedures usually included the removal of the telluric absorption lines through an interactive process (task telluric) using a spectrum of an early-type rapidly rotating star.

Suitable McDonald spectra of 13 RCBs were available for analysis. In several cases, spectra of the same star from different epochs were available. The stars are listed in Table 1. At VBO, spectra were obtained of UW Cen, a star inaccessible from the McDonald Observatory, and also of XX Cam, R CrB, RY Sgr, and VZ Sgr. The UW Cen spectrum was complemented by a CTIO spectrum from 1992 (see Asplund et al. 2000).

\section{F I LINES}

In the atmosphere of an RCB star, fluorine is present as neutral atoms. The leading lines of $F_{I}$ in optical spectra come from excited levels with lower excitation potentials of $12.7 \mathrm{eV}$ or higher. The $2 p^{4}\left({ }^{3} P\right) 3 s-2 p^{4}\left({ }^{3} P\right) 3 p$ transition array provides 27 lines from six multiplets with lower excitation potentials $12.70-13.02 \mathrm{eV}$. The adopted $g f$-values come from Musielok et al. (1999), who measured relative transition probabilities for lines in the $3 s-3 p$ (and $3 p-3 d$ ) array using emission lines from a wall-stabilized arc and used Opacity Project calculations of selected strong lines to place results on an absolute scale. The $g f$-values are estimated to be accurate to $15 \%-20 \%$.

\footnotetext{
${ }^{2}$ The IRAF software is distributed by the National Optical Astronomy Observatory under contract with the National Science Foundation.
}

The predicted equivalent widths $\left(W_{\lambda}\right)$ of $\mathrm{F}$ I in RCB stars are sensitive to the effective temperature $\left(T_{\text {eff }}\right)$. This sensitivity is highlighted with a simple calculation of the predicted $W_{\lambda}$ for the F I 26902.47 line, the fourth strongest line in the transition array, with a $g f$-value of 1.51 for models with a number density ratio $\mathrm{C} / \mathrm{He}=1 \%$. The $W_{\lambda}$ varies from $75 \mathrm{~m} \AA$ at $\left(T_{\text {eff }}, \log g\right)=$ $(8000 \mathrm{~K}, 1.0)$ to $35 \mathrm{~m} \AA$ at $(7000 \mathrm{~K}, 0.5)$ to $12 \mathrm{~m} \AA$ at $(6250 \mathrm{~K}$, $0.5)$ where the $\left(T_{\text {eff }}, \log g\right)$ combinations are representative of V3795 Sgr, VZ Sgr, and GU Sgr, respectively, and the F abundance chosen is representative of values reported for EHe stars (Pandey 2006). These predictions suggest that opportunities for detection of F I lines will be best in the hottest RCB stars. The high $T_{\text {eff }}$ sensitivity, perhaps suggested by the excitation potential of $13 \mathrm{eV}$, is moderated by the excitation potential (about 9$11 \mathrm{eV}$ ) of the high levels of the carbon atom providing the continuous opacity by photoionization. Note that the sensitivity of predicted $W_{\lambda}$ to $\log g$ at a given $T_{\text {eff }}$ is not appreciable. The calculations also show that the weakest lines in the transition array (say, $g f<0.3$ ) will not be detectable in cooler RCBs.

The following $\mathrm{F}_{\mathrm{I}}$ lines were identified as the principal or leading contributor to a stellar line: $7398.68,7754.69,6902.47$, 7425.65 , and $6834.26 \AA$. Figures 1 and 2 illustrate the windows that represent a couple of these F I lines, where the RCBs are ordered from top to bottom in order of decreasing effective temperature. All spectra are aligned to the rest wavelengths of wellknown lines that fall in the wavelength regions.

For all F I lines, a search was made for blending lines. Databases examined included the Kurucz database, ${ }^{3}$ tables of spectra of H, C, N, and O (Moore1993), Si (Moore 1965, 1967), and other elements (Moore 1972) Vienna Atomic Line Database (VALD), and the new Fe I multiplet table (Nave et al. 1994). The following remarks describe the quintet of useful lines in order to decreasing $g f$-value:

$7398.68 \AA(\log g f=+0.24)$. - This line is in an interorder gap for most McDonald spectra. The exceptions are McDonald spectra of XX Cam, UV Cas, and SU Tau and the VBT spectrum of UW Cen. The line is blended with the $\mathrm{N}_{\mathrm{I}} \lambda 7398.63$ line in

\footnotetext{
${ }^{3}$ See http://kurucz.harvard.edu.
} 


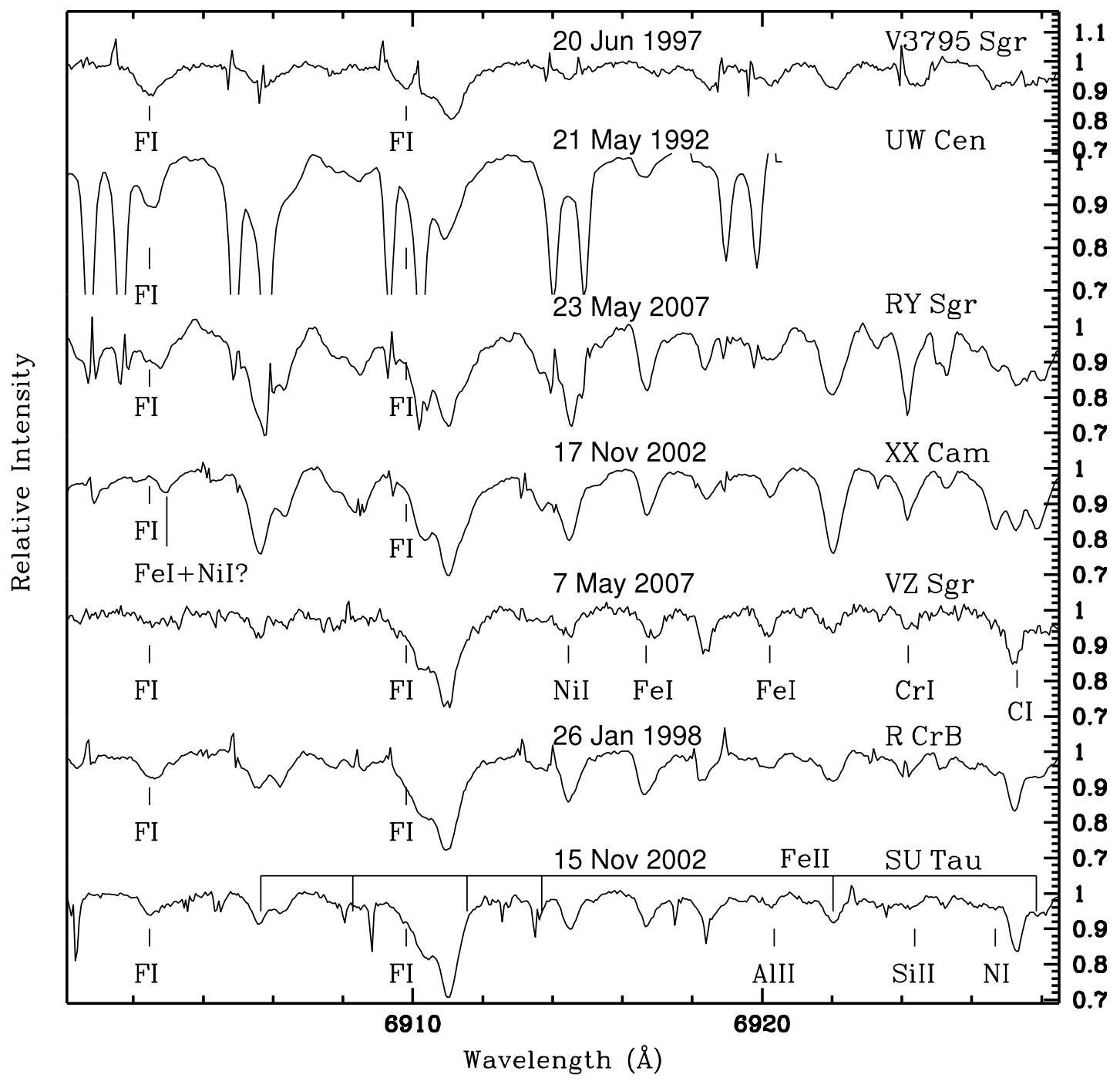

FIG. 1.- Spectral region from 6900-6928 A is shown for seven RCBs with the hottest star at the top and the coolest star at the bottom. The F I line at $6902.47 \AA$ labeled

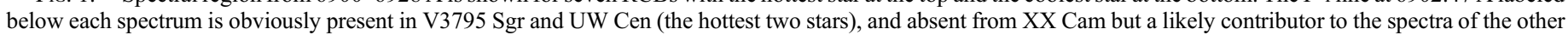

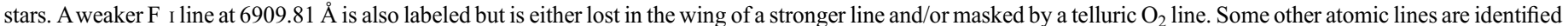
that apply to all spectra.

XX Cam, UV Cas, and SU Tau but the F I line's contribution is obtainable for UW Cen. The CN $(4,1) \lambda 7398.473$ may be contributing to the blue wing of this line for cooler RCBs.

$7754.69 \AA(\log g f=+0.24)$. - The major contributors to the red wing of this line are $\mathrm{Sm}$ II $27755.072, \mathrm{Mg}_{\mathrm{I}}$ 27755.236, and Fe II $\lambda 7755.563$. $\mathrm{CN}(5,2) 7753.767 \AA$ may be contributing to the blue wing of this line for cooler RCBs. The F I line's contribution for V3795 Sgr is measureable.

$6902.47 \AA(\log g f=+0.18)$. - This region is crossed by telluric absorption $\left(\mathrm{O}_{2}\right)$ lines. From the available database of RCB spectra at maximum light, only those spectra were used in which the $6902.47 \AA$ stellar feature avoids these telluric absorption lines (see Fig. 1). Also, further care has been taken to remove these telluric absorptions by ratioing these spectra with a spectrum of an earlytype rapidly rotating star. This line is present in V3795 Sgr and UW Cen with a depth comparable to that of the 27754.69 line of similar $g f$-value. There is obviously a line present to the red that dominates in XX Cam and other Fe-rich RCBs. The suspected lines are Ni I at $6902.791 \AA$ (VALD), and Fe I at $6902.956 \AA$ (Kurucz's database). Through spectrum synthesis (see $\S 4$ for details), the line provides an estimate of or an upper limit to the $\mathrm{F}$ abundance.
For the spectrum synthesis of the region, we take Fe I to be the significant blend in the RCB stars' spectra instead of the Ni I line. XX Cam's spectra clearly shows this Fe $\mathrm{I} 26902.956$ line with little or no contribution from the F I 26902.47 line (Fig. 1). We estimate the $g f$-value of this $\mathrm{Fe}$ I $\lambda 6902.956$ line by inversion using the XX Cam's spectrum; the Fe abundance is taken from Asplund et al. (2000). Figure 3 shows the spectrum synthesis of this $\mathrm{F}$ I line region for several RCBs.

$7425.64 \AA(\log g f=-0.19)$. - This line is detected in V3795 Sgr and UW Cen but not in other RCBs for which the upper limit to the $\mathrm{F}$ abundance is consistent with estimates from other lines.

$6834.26 \AA(\log g f=-0.21)$. - This line contributes in V3795 Sgr and UW Cen, and shows its presence in other RCBs (Figs. 2 and 4). This line is used for abundance determination after accounting for all the known blends. Blends include La II $\lambda 6834.009$, $\mathrm{CN}(7,3) \lambda 6834.236$, and $\mathrm{N}_{\mathrm{I}} \lambda 6834.074$. Kurucz's database also lists a La II $\lambda 6837.904$ line, which is about the same strength as the La II $\lambda 6834.009$ line. However, the La II $\lambda 6837.904$ line is not detected even in the $s$-process enriched RCBs. For the $\mathrm{N}_{\mathrm{I}}$ line, we take the $g f$-value from Kurucz's database. XX Cam's spectrum essentially has little or no contribution from the $\mathrm{F}_{\mathrm{I}} \lambda 6834.26$ line 


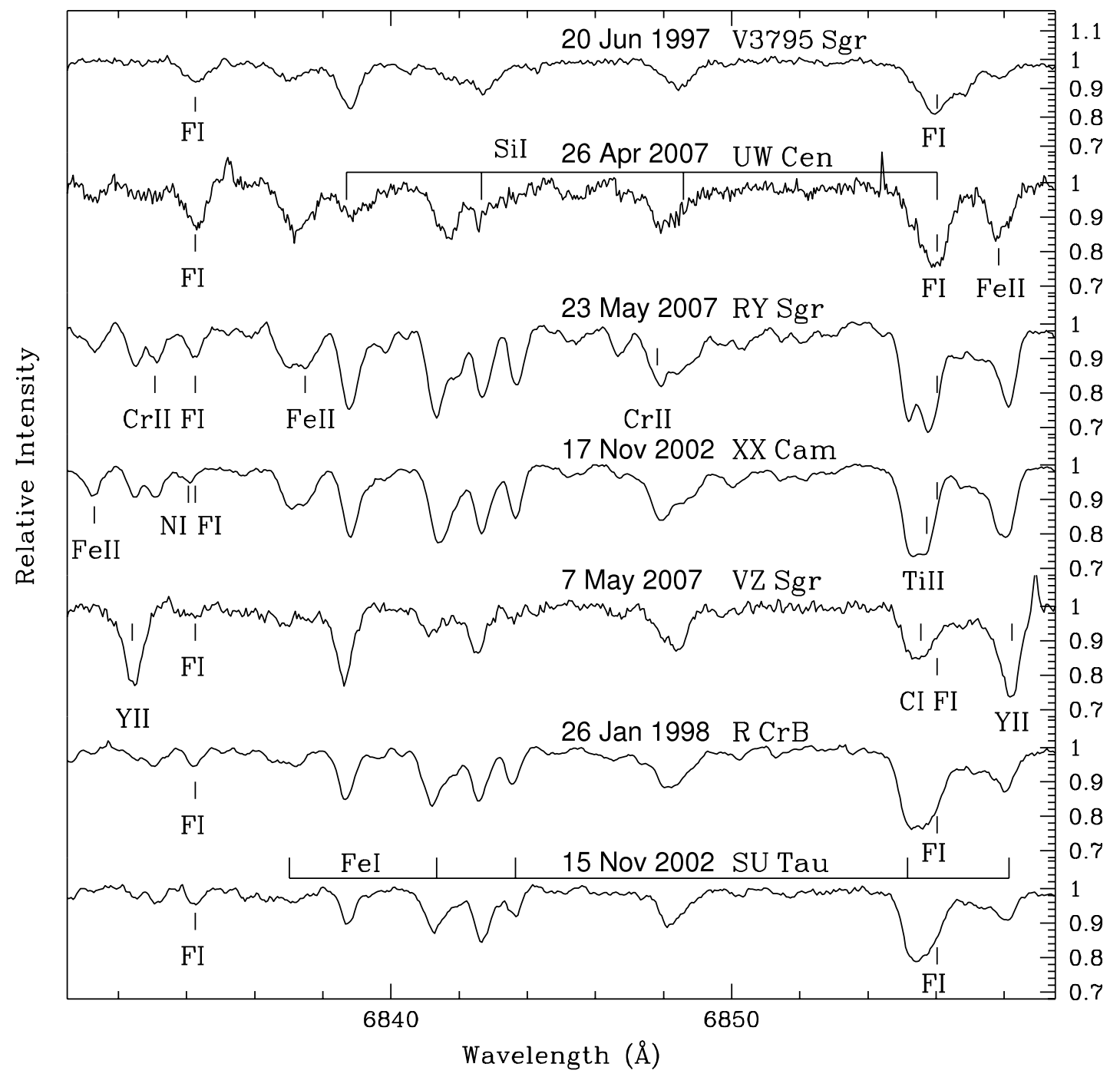

FIG. 2.- Spectral region from 6830-6860 $\AA$ is shown for seven RCBs with the hottest star at the top and the coolest star at the bottom. The F I line at $6834.26 \AA$ labeled below each spectrum is obviously present in V3795 Sgr and UW Cen (the hottest two stars), and absent from XX Cam but a likely contributor to the spectra of the other stars. A stronger F I line at $6856.03 \AA$ is also labeled. This line is clearly present for V3795 Sgr and UW Cen. In cooler stars, the F I contribution is blended severely with a Si I and a $\mathrm{Fe}$ I line. Some other atomic lines are identified that apply to all spectra.

(Fig. 2). The predicted N I profile at $6834.074 \AA$ A reproduces the observed profile for XX Cam; the $\mathrm{N}$ abundance is from Asplund et al. (2000). Also, the equivalent widths of the common N I lines measured in our RCB stars' spectra are in agreement with the Asplund et al. (2000) measurements within the expected uncertainties. We adopt the $\mathrm{N}$ abundances from Asplund et al. (2000) for the analyzed RCBs except for SU Tau's N abundance that is from Pandey et al. (2004a). Figure 4 shows the spectrum synthesis of this F I line region for several RCBs.

Where they contribute, $\mathrm{CN}$ red-system lines are included with their $g f$-values taken from Kurucz's database. Note that $\mathrm{CN}$ lines listed in Kurucz's database with predicted or extrapolated energies are not included in the spectrum synthesis. The $\mathrm{CN}$ strengths are determined from the strongest $\mathrm{CN}$ lines observed across the wavelength region of the analyzed RCBs. $\mathrm{CN}$ contributes to these $\mathrm{F}_{\mathrm{I}}$ lines only in the cooler members of the RCBs. Note that the $\mathrm{CN}(2,0)$ and $\mathrm{CN}(3,0)$ bandheads are clearly present in the cooler members of the RCBs; $\mathrm{CN}$ bands are typically strong in visible spectra of cool RCB stars (e.g., Clayton 1996 and references therein).

A search was made for all other lines in the transition array but with the exception of clear indications of a F I contribution for
V3795 Sgr and UW Cen, the F I line is masked by blending lines in the other RCBs.

\section{ABUNDANCE ANALYSIS}

The fluorine abundances were determined from the $\mathrm{F}$ I lines by adopting the procedure described in Pandey et al. (2006). Uppsala H-deficient model atmospheres (Asplund et al. 1997a) are used with the Uppsala spectral synthesis LTE code BSYNRUN to compute either the equivalent width of a F I line or a synthetic spectrum for a selected spectral window. The synthetic spectrum was convolved with a Gaussian profile to account for the combined effects of the stellar macroturbulence, rotational broadening, and the instrumental profile, before matching with the observed spectrum. The fact that several $\mathrm{F}$ I lines are blended with other lines requires that the fluorine abundance be extracted by spectrum synthesis. Although F I $\lambda 6902.47$ and F I $\lambda 6834.26$ lines are blended in most of the analyzed RCBs, we could account for the contributing blending lines and extract the F I component just from these lines. For the two hottest RCBs, fluorine was also measured from the F I 27425.64 and F I 27398.68 lines in UW Cen, and in V3795 Sgr from the F I 27425.64 and F I $\lambda 7754.69$ lines. The number of usable $\mathrm{F} \mathrm{I}$ lines declines from four 


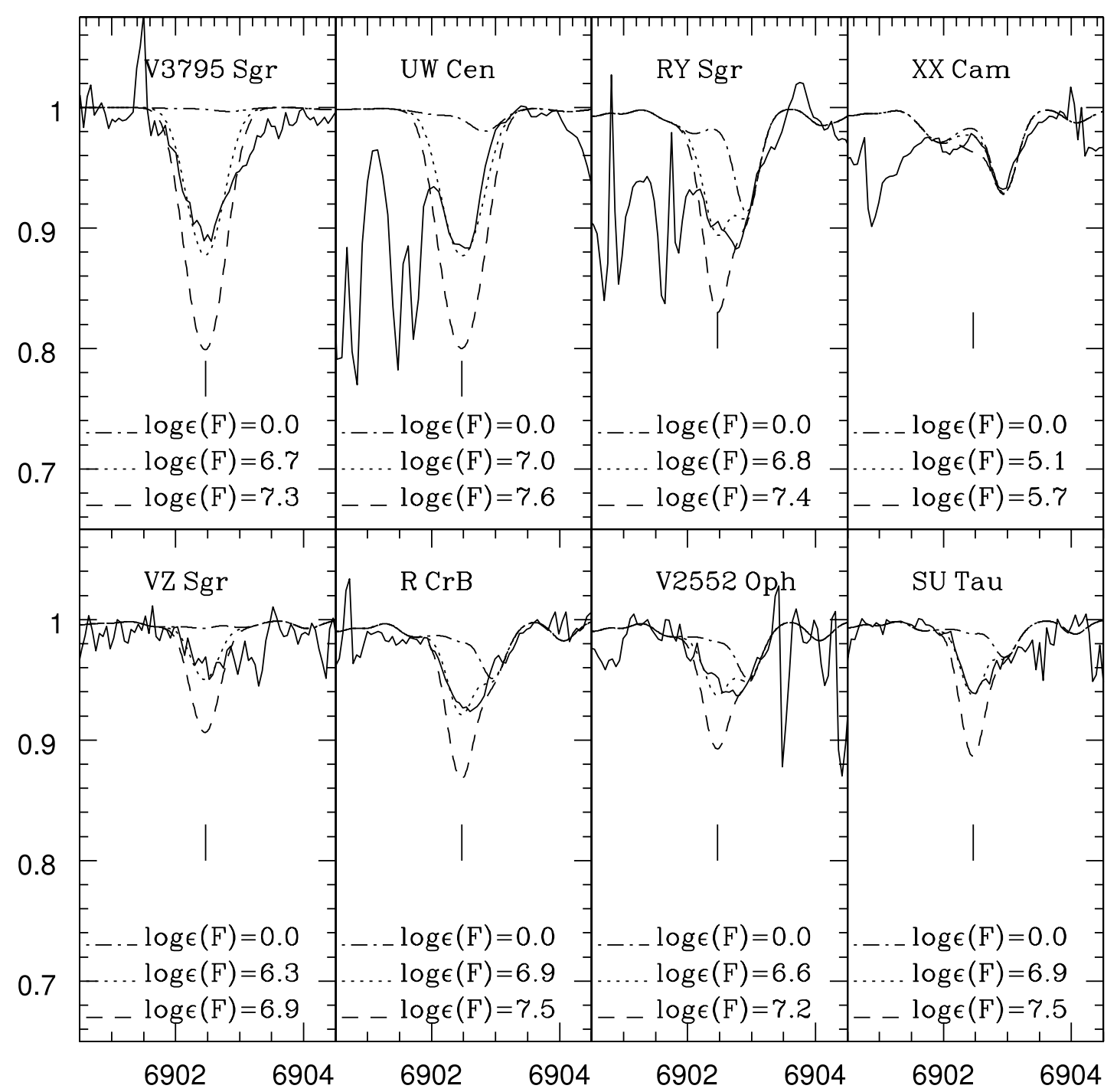

FIG. 3.-Observed F I 26902.47 line profiles (solid lines) of several RCB stars. Synthetic spectra are shown for three fluorine abundances, as shown on the figure.

for V3795 Sgr and UW Cen, the hottest RCBs, to two or zero in the coolest RCBs. This trend reflects the decline of the predicted equivalent width for constant $\mathrm{F}$ abundance with decreasing $T_{\text {eff }}$. As a reminder, we note that 11 to $17 \mathrm{~F}$ I lines were usable for the EHes with $T_{\text {eff }}$ 's from 8750 to $12,750 \mathrm{~K}$.

The adopted stellar parameters: effective temperature $\left(T_{\mathrm{eff}}\right)$, surface gravity $(\log g)$, and microturbulent velocity $(\xi)$, are from Asplund et al. (2000) for most of the analyzed RCBs. The stellar parameters adopted for V2552 Oph, and V CrA are from Rao \& Lambert (2003) and Rao \& Lambert (2007), respectively. We adopt the Uppsala model atmospheres (Asplund et al. 1997a) with a carbon-to-helium abundance ratio $\mathrm{C} / \mathrm{He}$ of $1 \%$ for the analyzed $\mathrm{RCBs}$. The $\mathrm{C} / \mathrm{He}$ ratio is not directly determinable for the $\mathrm{RCBs}$. The choice of $\mathrm{C} / \mathrm{He}$ of $1 \%$ is provided by the measured $\mathrm{C} / \mathrm{He}$ of EHe stars, the close relatives of RCBs. The analyzed EHe stars have a mean $\mathrm{C} / \mathrm{He}$ of $0.8 \%$ (Pandey et al. 2006 and references therein; Pandey \& Reddy 2006), which suggests that the adopted $\mathrm{C} / \mathrm{He}$ ratio of $1 \%$ for the Uppsala model atmospheres is a reasonable choice.

For several of the analyzed RCBs, the best-fitting profiles for two of the studied F I wavelength regions are illustrated in Figures 3 and 4 . The measured mean fluorine abundances (Table 1) are given as $\log \epsilon(\mathrm{F})$, normalized such that $\log \Sigma \mu_{i} \epsilon(i)=12.15$, where $\mu_{i}$ is the atomic weight of element $i$.
The errors of the derived $\mathrm{F}$ abundances given in Table 1 are the line-to-line scatter. The number of lines used is given within brackets. The F abundances derived for the RCBs cooler or about effective temperature $6500 \mathrm{~K}$ are uncertain and are marked with a colon in Table 1. However, the F abundances so marked are determinations and not upper limits. An upper limit to the F abundance for XX Cam, V854 Cen, and the Sakurai's object is obtained by comparing the synthetic and the observed profiles of the $\mathrm{F}_{\mathrm{I}}$ line at $6834.26 \AA$ (Table 1). The effective error in $\mathrm{F}$ abundances due to uncertainty in the adopted stellar parameters, which are typically $\Delta T_{\text {eff }}= \pm 250 \mathrm{~K}, \Delta \log g= \pm 0.5 \mathrm{cgs}$, and $\Delta \xi=$ $\pm 2 \mathrm{~km} \mathrm{~s}^{-1}$, is about $0.3 \mathrm{dex}$. These uncertainties are similar across the sample of analyzed RCBs. Note that the derived fluorine abundances in LTE represent only the first step defining the absolute $\mathrm{F}$ abundance in these stars; more reliable values should, in principle, come from full non-LTE calculations.

A spectrum of Sakurai's object (V4334 Sgr), a final He-shell flash product, obtained on 1996 May 5 was available for analysis. The nondetection of the F I line at $6834.26 \AA$ provides with an upper limit to the $\mathrm{F}$ abundance (Fig. 5). Other potential F I lines in this (then) quite hot star were blended or in interorder gaps, e.g., the $\lambda 6902$ line is masked by overlying telluric lines. Note that the upper limit to the F abundance for the Sakurai's object in Table 1 is for the model's adopted $\mathrm{C} / \mathrm{He}$ ratio of $1 \%$; 


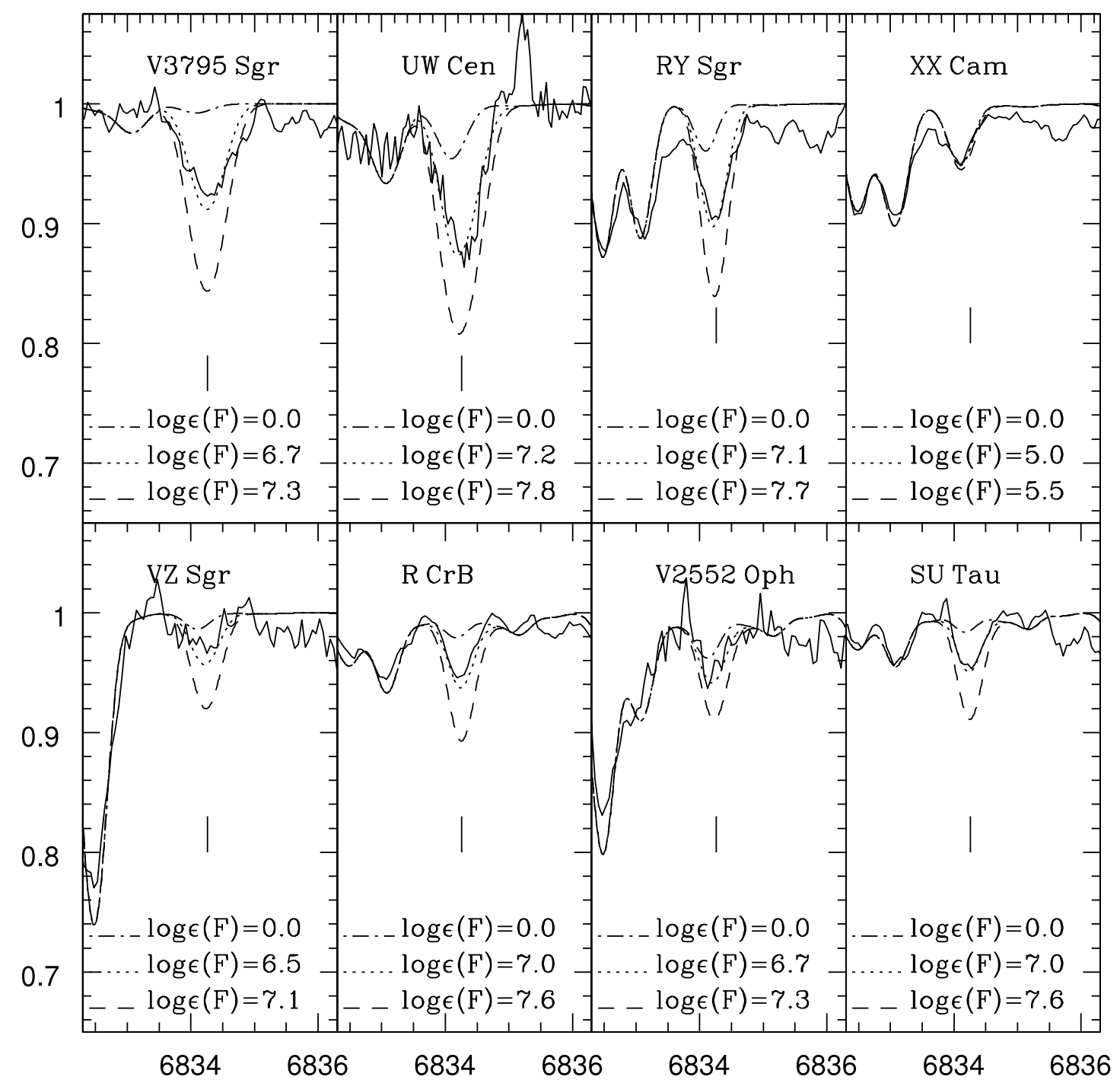

FIG. 4.- Observed F I $\lambda 6834.26$ line profiles (solid lines) of several RCB stars. Synthetic spectra are shown for three fluorine abundances, as shown on the figure.

model for a $\mathrm{C} / \mathrm{He}$ ratio of $10 \%$ returns an upper limit to the $\mathrm{F}$ abundance of about 6.4 dex. The stellar parameters are from Asplund et al. (1997b).

\section{DISCUSSION}

The analyzed RCBs (excluding stars with upper limits to the F abundances and, uncertain $\mathrm{F}$ abundances) have a mean $\mathrm{F}$ abundance of 6.7 which is the same for the analyzed EHes (Pandey 2006). Thus, the $F$ abundances are similar across an effective temperature range from about $6500-14,000 \mathrm{~K}$, an indication that non-LTE effects are possibly small. The $\mathrm{F}$ abundances of the analyzed RCB and EHe stars show no obvious trend with their abundances of other elements. In Figure 6 the F abundance is shown as a function of the $\mathrm{Fe}$ abundance for the RCBs from Table 1 and the EHes from Pandey (2006). The F abundances from RCBs and EHes are in good agreement and independent or almost so of the $\mathrm{Fe}$ abundance which spans a range of nearly two dex. In contrast, the $\mathrm{N}$ abundance is well correlated with the $\mathrm{Fe}$ abundance: the $\mathrm{N}$ abundances are consistent with the sum of the initial $\mathrm{C}, \mathrm{N}$, and $\mathrm{O}$ abundances suggesting that $\mathrm{CNO}-\mathrm{cycling}$ has controlled the $\mathrm{N}$ abundances. Among EHes and RCBs, the $\mathrm{O}$ abundance shows a significant scatter at a given $\mathrm{Fe}$ abundance. In Figure 7 the $\mathrm{F}$ abundance is shown as a function of the $\mathrm{O}$ abundance: the $\mathrm{F}$ abundances are uncorrelated with the $\mathrm{O}$ abundance.

\subsection{The Setting}

Knowledge of fluorine abundance in astronomical objects is limited but, nonetheless, helpful in interpreting these measurements for the RCBs (this paper) and EHes (Pandey 2006). The solar system abundance as measured from meteorites is $\log \epsilon(\mathrm{F})=$ $4.46 \pm 0.06$ (Lodders 2003; Asplund et al. 2005) with rather imprecise confirmation from the detection of HF lines in sunspots. Measurements of HF lines in K and M Galactic giants (Jorissen et al. 1992; Cunha et al. 2003) give a mean F abundance of $\log \epsilon(\mathrm{F})=4.62 \pm 0.12$ for a sample with a mean Fe abundance $([\mathrm{Fe} / \mathrm{H}]=-0.10 \pm 0.16)$ not significantly different from solar. The trend of $\mathrm{F}$ with $\mathrm{Fe}$ is quite uncertain. The sole Galactic star with a (slightly) subsolar Fe abundance is Arcturus with $[\mathrm{Fe} / \mathrm{H}]=$ -0.6 and a $\mathrm{F}$ abundance $[\log \epsilon(\mathrm{F})=4.10$; Cunha et al. 2003], suggesting that $[\mathrm{F} / \mathrm{Fe}] \sim 0$, but observations of additional metalpoor giants are required to confirm this hint.

The $\mathrm{F}$ versus $\mathrm{Fe}$ trend is one important datum for inferring the RCBs initial $\mathrm{F}$ abundance from their present $\mathrm{Fe}$ abundances. The mean Fe abundance from Asplund et al. (2000) and Rao \& Lambert (2003) for 14 majority RCBs is $\log \epsilon(\mathrm{Fe})=6.5$ with a 


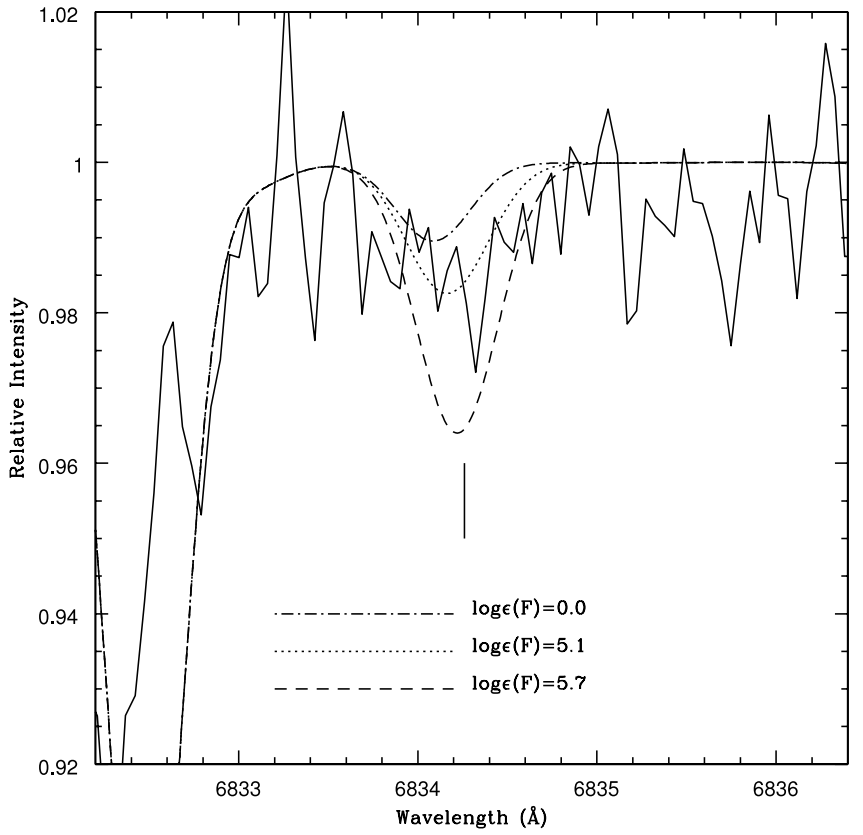

FIG. 5.-Observed F I 26834.26 line profile (solid line) of Sakurai's object. Synthetic spectra are shown for three fluorine abundances, as shown on the figure.

spread of 0.8 dex; three minority RCBs (V CrA, VZ Sgr, and V3795 Sgr) of very low Fe abundance are not included here; however, these three minority RCBs are not different in F abundances when compared with the majority RCBs. To this mean $\mathrm{Fe}$ abundance must be attached the qualification that the analyses assume a $\mathrm{C} / \mathrm{He}$ ratio of $1 \%$ by number but the analyses do not return the input $\mathrm{C}$ abundance but a value about 0.6 dex lower, a discrepancy termed "the carbon problem" by Asplund et al. Analyses of EHe stars (Pandey et al. 2006 and references therein), which are immune to the carbon problem, give a mean

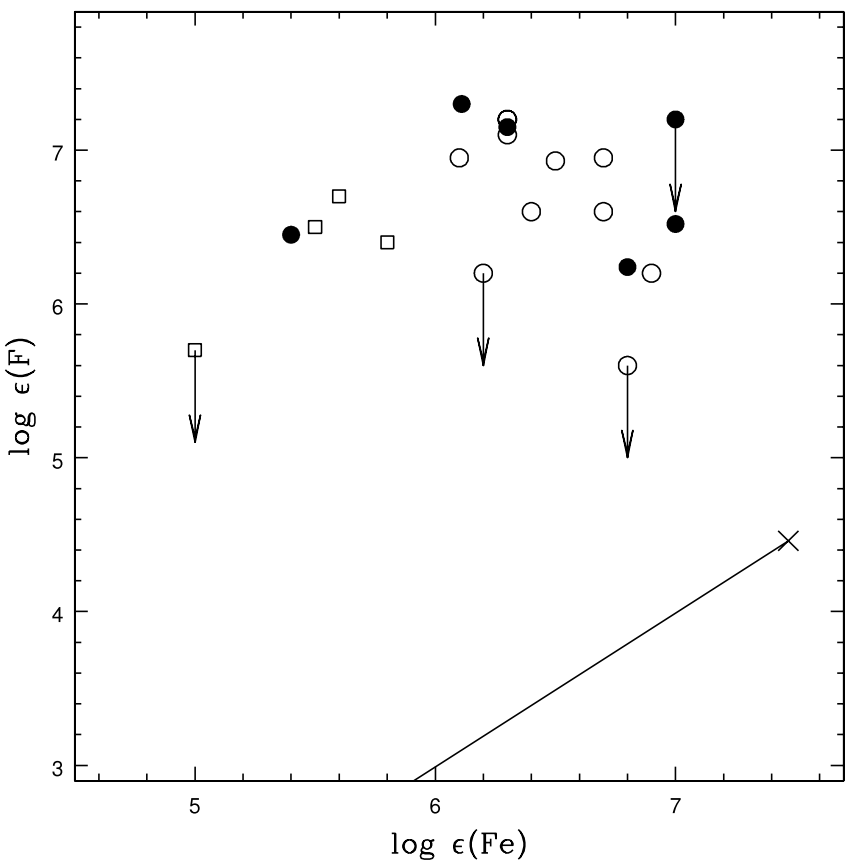

FIG. 6.-F abundance for majority RCBs (open circles), minority RCBs (open squares), and EHes (filled circles) as a function of the Fe abundance. The solid line shows the possible initial $\mathrm{F}$ abundances assuming the solar $\mathrm{F}$ and $\mathrm{Fe}$ abundances (large cross) and $[\mathrm{F} / \mathrm{Fe}]=0$.

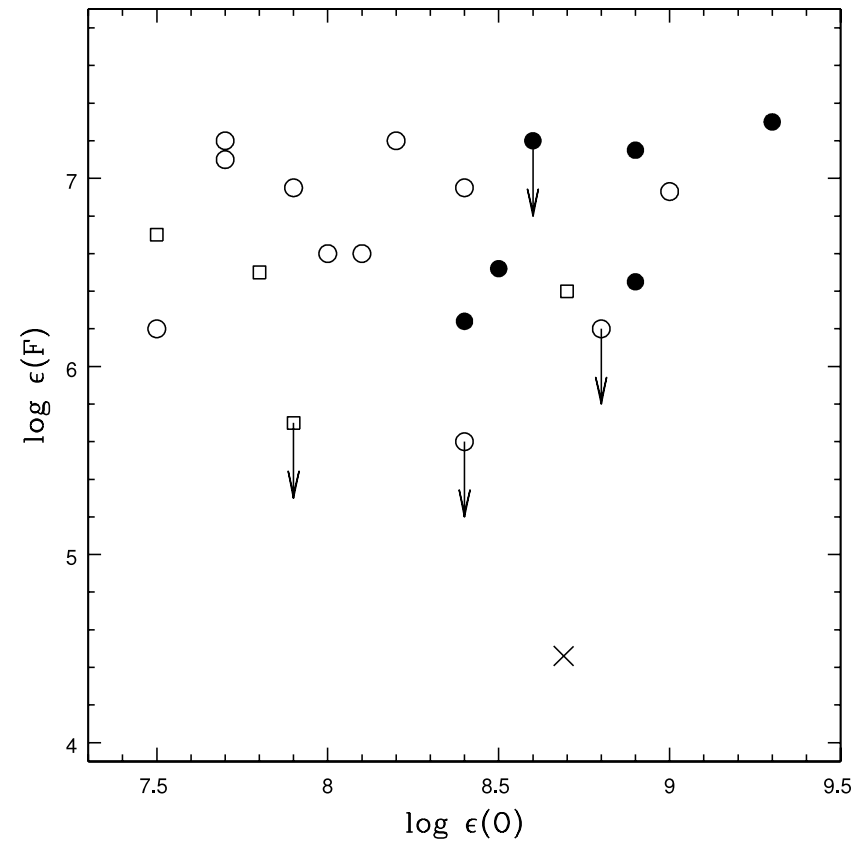

FIG. 7.-F abundance for majority RCBs (open circles), minority RCBs (open squares), and EHes ( filled circles) as a function of the $\mathrm{O}$ abundance. Solar $\mathrm{F}$ and $\mathrm{O}$ abundances are represented by the large cross.

Fe abundance $\log \epsilon(\mathrm{Fe})=6.7$ and a spread of 1.1 dex with two minority EHes excluded. The similar Fe abundances of EHes and RCBs suggests that the carbon problem is not adversely affecting the determination of the $\mathrm{Fe}$ abundance. The mean $\mathrm{C} / \mathrm{He}$ ratio found for the EHes is slightly less than the assumed $\mathrm{C} / \mathrm{He}=$ $1 \%$ for the RCBs. Adoption of the $\mathrm{C} / \mathrm{He}$ ratio of the EHes would reduce the mean $\mathrm{Fe}$ abundance for the RCBs by about 0.15 dex. The assumption is made that the Fe abundance is not affected by the stars' evolutionary history. It is striking that the EHes and RCBs are quite Fe-poor relative to the Sun. This might occasion concerns that the initial iron abundance has been reduced in the process of evolution to a H-poor star. In this connection, we note that abundance ratios $[\mathrm{X} / \mathrm{Fe}]$, especially those of the $\alpha$-elements$\mathrm{Mg}, \mathrm{Si}, \mathrm{S}$ and $\mathrm{Ca}$ - are those expected of metal-poor stars and not those of solar abundance stars (Asplund et al. 2000; Pandey et al. 2006). In the following discussion, we assume that the present abundances of iron are the initial values.

Although, as noted above, the observational data are sparse in the extreme, it seems safe to assume that $[\mathrm{F} / \mathrm{Fe}] \leq 0$ for metal-poor $([\mathrm{Fe} / \mathrm{H}]<0)$ stars. Galactic chemical evolution models confirm this expectation (Renda et al. 2004). In estimating the F enrichment for EHes and RCBs, we assume $[\mathrm{F} / \mathrm{Fe}]=0$ and the solar $\mathrm{F}$ abundance $\log \epsilon(\mathrm{F})=4.46$. Thus, a representative $\mathrm{RCB}$ with $\log \epsilon(\mathrm{Fe})=6.5$ or $[\mathrm{Fe} / \mathrm{H}]=-1.0$ is assumed to have an initial $\mathrm{F}$ abundance of $\log \epsilon(\mathrm{F}) \leq 3.5$. More interestingly, the $\mathrm{F}$ overabundances are extremely large: enhancements of about 800 , 2500 , and 8000 at $\log \epsilon(\mathrm{Fe})=7.5,6.5$, and 5.5, respectively. The fact that the overabundances are very large is qualitatively true irrespective of all plausible values for $[\mathrm{F} / \mathrm{Fe}]$ as a function of $[\mathrm{Fe} / \mathrm{H}]$.

\subsection{The DD Scenario and Fluorine}

In the "cold" (i.e., no nucleosynthesis during the merger) version of the DD scenario, the He white dwarf is stripped and accreted by the $\mathrm{C}-\mathrm{O}$ white dwarf. During the active phase of the merger, the accreted material is mixed with the outer layer of the $\mathrm{C}-\mathrm{O}$ white dwarf, which is the residue of the He-intershell of an 
AGB star for which the $\mathrm{C}-\mathrm{O}$ white dwarf was the electron degenerate core. Mixing may also penetrate the outer layers of the $\mathrm{C}-\mathrm{O}$ core. In the "hot" DD scenario, nucleosynthesis occurs during accretion. Following both cold and hot mergers, the hot luminous H-poor star evolves to the red before returning to the blue and descending the white dwarf cooling track. Along this track, He-shell flashes are likely to occur and dredge-ups are likely to alter the surface chemical evolution. These alterations have not been extensively studied theoretically. Limited predictions for a slow rate of accretion have been reported by Saio \& Jeffery (2002).

Given the principal features of the expected compositions of the He white dwarf and the He-shell around the C-O white dwarf, one simple recipe that provides an atmosphere with a RCB- and EHe-like composition is as follows: a $0.3 M_{\odot}$ He white dwarf is mixed with $0.03 M_{\odot}$ of He-intershell material. This cold recipe does not consider nucleosynthesis to occur during or following the merger. The relative masses of He-intershell and He white dwarf determine the final $\mathrm{C} / \mathrm{He}$; the $\mathrm{He}$ white dwarf is rich in $\mathrm{He}$ but highly deficient in $\mathrm{C}$ and $\mathrm{O}$ and the He-intershell is relatively rich in $\mathrm{C}$ and $\mathrm{O}$. The $\mathrm{N}$ abundance is provided primarily by the He white dwarf. A H-rich surface layer on the He and/or the $\mathrm{C}-\mathrm{O}$ white dwarf provides the residual $\mathrm{H}$ for the RCB and $\mathrm{EHe}$. For discussion of such a recipe and how it may match observed compositions of RCBs and EHes, see Saio \& Jeffery (2002) and Pandey et al. (2006).

For elements greatly enriched in the He-intershell, their abundance in the RCB and EHe is diluted by the material from the He white dwarf. A dilution by a factor of order 10 is necessary in light of the expected abundances of $\mathrm{C}$ and $\mathrm{He}$ in the He-intershell. In the case of $\mathrm{F}$, we may appeal to theoretical predictions of $\mathrm{F}$ abundances in the He-intershell of AGB stars (Lugaro et al. 2004) and to observations of F in PG 1159 stars. PG 1159 stars are H-deficient very hot $\left(T_{\text {eff }}=75,000-200,000 \mathrm{~K}\right)$ post-AGB stars whose surfaces "offer the unique possibility of studying intershell abundances directly" (Werner \& Herwig 2006). The PG 1159 stars are considered to be products of the FF scenario and to involve a "very late thermal pulse" (VLTP; see Werner \& Herwig [2006] for the difference between a very late thermal pulse and a late thermal pulse). The $\mathrm{C} / \mathrm{He}$ ratio of PG 1159 stars is too high for EHe and RCB stars to be identified with the FF scenario. In the DD scenario, the high $\mathrm{C} / \mathrm{He}$ ratio is diluted to a lower ratio when the FF-like layer of the $\mathrm{C}-\mathrm{O}$ white dwarf is mixed with the He white dwarf.

Fluorine in PG 1159 stars shows a range of abundances (Werner et al. 2005; Werner \& Herwig 2006) from solar to 250 times solar. As Werner \& Herwig discuss, this range is not out of line with theoretical predictions for the He-intershell. In addition, the theoretical predictions account fairly well for the observed enrichments of $\mathrm{F}$ in ( $\mathrm{H}$ normal) giant stars known to be affected by the third dredge-up that brings material from the He-intershell into the envelope (Jorissen et al. 1992). But in the cold DD scenario, the He-intershell material is diluted, according to the canonical recipe, by a factor of about 10 and, then, overabundances of up to 25 times solar for the RCBs and EHes are predicted. The observed overabundances of $\mathrm{F}$ range upward of 1000 times. Unless the VLTP experienced by the PG 1159 stars drastically reduced the $\mathrm{F}$ abundance of the He-intershell or the nucleosynthesis experienced by the He-intershell of the C-O white dwarf differs markedly from that experienced by the PG 1159 stars, it would appear that the cold DD scenario cannot account for the observed $\mathrm{F}$ abundances of RCBs and EHes.

The upper limit for the F abundance of Sakurai's object is consistent with but does not demand ingestion of $\mathrm{H}$ and the final
He-shell flash leading to a complete conversion of ${ }^{14} \mathrm{~N}$ to ${ }^{22} \mathrm{Ne}$. The temperatures are so high that ${ }^{18} \mathrm{O}$, which is the seed for ${ }^{19} \mathrm{~F}$, is destroyed. Note that the infrared spectra of Sakurai's object obtained during 1997-1998 when it had strong CO overtone bands showed no evidence for ${ }^{12} \mathrm{C}^{18} \mathrm{O}$ (Geballe et al. 2002). Therefore, Sakurai's object and the other FF stars, when compared with most of the RCB and HdC stars, probably have different origins.

A "hot" DD scenario is fuelled by a rapid deposition of accreted He-rich material on the surface of the C-O white dwarf. Observational evidence that suggests a hot DD scenario is provided by Clayton et al.'s $(2005,2007)$ discovery of huge overabundances of ${ }^{18} \mathrm{O}$ in $\mathrm{HdC}$ and some cool RCBs through medium-resolution spectroscopy of the first-overtone vibration-rotation bands of the CO molecule near $2.3 \mu \mathrm{m}$. High-resolution spectroscopy confirms that ${ }^{16} \mathrm{O} /{ }^{18} \mathrm{O}$ ratios are low: García-Hernández et al. (2007) find ${ }^{16} \mathrm{O} /{ }^{18} \mathrm{O}=0.3-0.5$ for the three $\mathrm{HdCs}$ with $\mathrm{CO}$ bands and 16 for the cool RCB S Aps.

Clayton et al. (2007) show via qualitative arguments about temperature, density, and timescale that the merger leads to temperatures at the base of the accreted material approaching 200 million $\mathrm{K}$ with conditions ripe for nucleosynthesis lasting several years. Their calculations with a reaction network show that the plentiful supply of ${ }^{14} \mathrm{~N}$ in the He white dwarf may be converted to ${ }^{18} \mathrm{O}$ by $\alpha$-capture. Without this conversion, the DD scenario cannot account for the ${ }^{18} \mathrm{O}$ overabundances; the expected ${ }^{18} \mathrm{O}$ abundance in the He white dwarf and in the $\mathrm{He}$-intershell of the $\mathrm{C}-\mathrm{O}$ white dwarf are very low. The present measurements of ${ }^{18} \mathrm{O}$ in $\mathrm{HdC}$ and RCB stars indicate that no more than a minor fraction of the ${ }^{14} \mathrm{~N}$ is converted to ${ }^{18} \mathrm{O}$. ("No overproduction of ${ }^{18} \mathrm{O}$ is expected in the FF scenario," state Clayton et al. [2007].)

The possibilities for nucleosynthesis in the hot DD scenario are more extensive if the He white dwarf has a H-rich outer layer. Since many $\mathrm{HdC}, \mathrm{RCB}$, and EHe stars have retained some $\mathrm{H}, \mathrm{H}$ was likely a participant in the nucleosynthesis. Clayton et al. (2007) note that the presence of protons in ${ }^{18} \mathrm{O}$-rich gas promotes the synthesis of ${ }^{19} \mathrm{~F}$. The reaction ${ }^{18} \mathrm{O}(p, \gamma){ }^{19} \mathrm{~F}$ provides ${ }^{19} \mathrm{~F}$ directly. The ${ }^{18} \mathrm{O}$ is converted about 150 times faster by proton captures to ${ }^{12} \mathrm{C}:{ }^{18} \mathrm{O}(p, \alpha){ }^{15} \mathrm{~N}(p, \alpha){ }^{12} \mathrm{C}$. Clayton et al.'s $(2007$, their Fig. 6) sample single-zone calculation for a mixture with $\mathrm{H}$ added and for an arbitrary temperature history and initial composition showing effects of some He-burning shows that the ${ }^{19} \mathrm{~F}$ abundance increased by about a dex at the cessation of nucleosynthesis from an initial value about a factor of three below solar to a factor of two above solar. At its peak, $\mathrm{F}$ was briefly about 100 times above its solar abundance before ${ }^{19} \mathrm{~F}(\alpha, p){ }^{22} \mathrm{Ne}$ took its toll. Clearly, although ${ }^{19} \mathrm{~F}$ synthesis is demonstrated by these calculations, a remaining challenge is to show that the parameter space covered by the hot DD scenario includes the possibility of robustly increasing the $\mathrm{F}$ abundance not by a factor of 100 but to the observed levels of 1000-8000 times over solar across the metallicity range $[\mathrm{Fe} / \mathrm{H}]$ of -0.5 to -2.0 .

Fluorine abundances appear to demand that $\mathrm{H}$ be a key ingredient in the hot DD scenario. This is suggested by the $\mathrm{F} / \mathrm{N}$ ratios. The $\mathrm{N}$ abundances of the EHe stars are equal to the sum of the initial $\mathrm{C}, \mathrm{N}$, and $\mathrm{O}$ abundances, as expected for He-rich material exposed to CNO-cycling (Pandey et al. 2006). The RCBs give a similar result. The observed $\mathrm{N} / \mathrm{F}$ ratios are about 80,60 , and 5 at $[\mathrm{Fe} / \mathrm{H}]=-0.5,-1.0$, and -2.0 , respectively. Given that about 150 times more ${ }^{18} \mathrm{O}$ is diverted to ${ }^{12} \mathrm{C}$ than is converted to ${ }^{19} \mathrm{~F}$, the observed N/F abundances imply that total conversion of the ${ }^{14} \mathrm{~N}$ from the He white dwarf to ${ }^{18} \mathrm{O}$ cannot provide the observed ${ }^{19} \mathrm{~F}$ abundances and this total conversion certainly cannot account for the fact that the ${ }^{14} \mathrm{~N}$ across the sample of EHe and 
RCB stars is consistently close to that expected from CNO-cycling for the He white dwarf. The conclusion seems to be that hot accretion must involve addition of $\mathrm{H}$ to the extent that $\mathrm{N}$ and ${ }^{18} \mathrm{O}$ are regenerated by H-burning. This and other nucleosynthetic consequences are illustrated by Clayton et al. (2007, their Fig. 6). Their calculation if representative would explain the star-to-star variation in $\mathrm{O}$ abundances but the fact that the observed abundances are close to the sum of the initial $\mathrm{C}, \mathrm{N}$, and $\mathrm{O}$ abundances has then to be regarded as fortuitous. Obviously, challenges remain in accounting for the $\mathrm{F}$ abundances. One challenge arises from incorporating Clayton et al.'s single-zone calculations into a full model of the accreting star and its subsequent evolution with attendant shell flashes and dredge-ups.

Another challenge involving nucleosynthesis concerns the abundance of Li. Lithium is present in four of the analyzed 15 majority RCBs (Lambert \& Rao 1994) and in one of the five HdCs (Rao \& Lambert 1996) with an appreciable abundance $\log \epsilon(\mathrm{Li}) \sim 3$. The only conceivable reservoir of $\mathrm{Li}$ in the two white dwarfs ahead of their merger is the H-rich layer around the He white dwarf but the mass of this is too small to result in the above $\mathrm{Li}$ abundances, even if the H-rich layer had retained its initial $\mathrm{Li}$ abundance. Lithium synthesis is presumed to involve ${ }^{3} \mathrm{He}$ and the reactions ${ }^{3} \mathrm{He}(\alpha, \gamma){ }^{7} \mathrm{Be}(e, \nu){ }^{7} \mathrm{Li}$. The ${ }^{3} \mathrm{He}$ may be present in the H-rich layer as the product of the first steps of the $p-p$ chain and as an initial ingredient of the star. In a hot DD scenario in which ${ }^{14} \mathrm{~N}$ is converted to ${ }^{18} \mathrm{O}$, it seems inconceivable that ${ }^{7} \mathrm{Be}$ or ${ }^{7} \mathrm{Li}$ can avoid thorough destruction by $\alpha$-capture; enrichment of $\mathrm{Li}$ in parallel with enrichment of ${ }^{18} \mathrm{O}$ and $\mathrm{F}$ seems impossible in nucleosynthesis accompanying the DD scenario (Clayton et al. 2007). One supposes that Li may be synthesized in "warm" DD scenarios in which mild nucleosynthesis occurs with temperatures too low for ${ }^{18} \mathrm{O}$ production. Lithium synthesis is possible in the FF scenario. Most irritatingly, the $\mathrm{HdC}$ and $\mathrm{RCB}$ stars for which ${ }^{18} \mathrm{O}$ has been detected are not those in which $\mathrm{Li}$ is present; note that the possibility of ${ }^{18} \mathrm{O}$ enrichment in the Li-rich HdC star HD 148839 is not discarded by GarcíaHernández et al. (2007). However, the Li-rich RCB stars are not distinguishable from other RCBs and all have the $\mathrm{He} / \mathrm{C} / \mathrm{N} / \mathrm{O}$ ratios of a DD scenario and incompatible with a FF scenario. It is intriguing that the Li-rich stars (UW Cen, R CrB, and SU Tau) are among the stars with the highest $\mathrm{F}$ abundances. The detection of high $\mathrm{F}$ in both minority and majority RCBs is also very interesting since ${ }^{18} \mathrm{O}$ observations do not include any of these stars as these are too hot. This adds the minority RCBs as well as the Li-rich RCB stars to the stars that might have large amounts of ${ }^{18} \mathrm{O}$.

\section{CONCLUDING REMARKS}

Lithium and fluorine are not the sole or even the principal concerns for the DD scenario. One wonders why the $\mathrm{C} / \mathrm{He} \sim 0.01$ ratio, as measured for the EHes, spans a narrow range. The He is contributed primarily by the He white dwarf and the $\mathrm{C}$ by the Heintershell of the C-O white dwarf. What sets the mass ratio of these two regions rather precisely?

The evolutionary connections between the H-deficient stars remains unproven but with obvious potential links. Both possible sequences, $\mathrm{HdC} \rightarrow \mathrm{RCB} \rightarrow \mathrm{EHe}$ and $\mathrm{EHe} \rightarrow \mathrm{RCB} \rightarrow \mathrm{HdC}$, are possible because the DD scenario leads to a very hot star evolving to lower temperatures before the evolutionary track reverses direction. An extension $\mathrm{EHe} \rightarrow \mathrm{SdO} \rightarrow \mathrm{O}(\mathrm{He})$ to higher temperatures and higher surface gravities seems plausible (Rauch et al. 2006; Werner \& Herwig 2006). The stars in this sequence are all candidates for an origin via the DD scenario, i.e., they have the low $\mathrm{C} / \mathrm{He}$ ratio and not the high ratio characteristic of the $\mathrm{FF}$ scenario; a similar sequence for the FF scenario exists linking objects like FG Sge and V4334 Sgr (Sakurai's object) through the $[\mathrm{WC}]$ to the PG1159 stars.

Continued study of the compositions of stars along these sequences will serve to sharpen understanding of the evolutionary links and the entry points for stars into the sequence. New data such as that for $\mathrm{F}$ and ${ }^{18} \mathrm{O}$ stimulate continued study. The $\mathrm{F}$ abundances of RCB and EHe stars are quite similar in value and spread and thus support the presumed evolution from RCB to EHe. On the other hand, there is a hint that the ${ }^{16} \mathrm{O} /{ }^{18} \mathrm{O}$ ratios of the HdC and RCB stars may be different with the ratio being systematically higher in the (cool) RCBs than in the three HdCs. Is the difference merely a distraction caused by small number statistics or is it symptomatic of a change introduced as a $\mathrm{HdC}$ evolves to a cool RCB or does it reflect that the DD scenario can place stars on the evolutionary sequence at different starting points with different compositions?

We thank the referee Geoff Clayton for the nice report. G. P. thanks the VBT staff for their assistance. This research has been supported in part by the Robert A. Welch Foundation of Houston, Texas.
Asplund, M., Grevesse, N., \& Sauval, A. J. 2005, in ASP Conf. Ser. 336, Cosmic Abundances as Recods of Stellar Evolution and Nocleosynthesis, ed. T. G. Barnes III \& F. N. Bash (San Francisco: ASP), 25

Asplund, M., Gustafsson, B., Kiselman, D., \& Eriksson, K. 1997a, A\&A, 318, 521

Asplund, M., Gustafsson, B., Lambert, D. L., \& Rao, N. K. 1997b, A\&A, 321, L17

. 2000, A\&A, 353, 287

Clayton, G. C. 1996, PASP, 108, 225

Clayton, G. C., Geballe, T. R., Herwig, F., Fryer, C., \& Asplund, M. 2007, ApJ, 662,1220

Clayton, G. C., Herwig, F., Geballe, T. R., Asplund, M., Tenenbaum, E. D., Engelbracht, C. W., \& Gordon, K. D. 2005, ApJ, 623, L141

Cunha, K., Smith, V. V., Lambert, D. L., \& Hinkle, K. H. 2003, AJ, 126, 1305

García-Hernández, D. A., Hinkle, K. H., Lambert, D. L., \& Eriksson, K. 2007, ApJ, submitted

Geballe, T. R., Evans, A., Smalley, B., Tyne, V. H., \& Eyres, S. P. S. 2002, Ap\&SS, 279, 39

Jorissen, A., Smith, V. V., \& Lambert, D. L. 1992, A\&A, 261, 164

Lambert, D. L., \& Rao, N. K. 1994, J. Astrophys. Astron., 15, 47

Lodders, K. 2003, ApJ, 591, 1220
Lugaro, M., Ugalde, C., Karakas, A. I., Görres, J., Wiescher, M., Lattanzio, J. C., \& Cannon, R. C. 2004, ApJ, 615, 934

Moore, C. E. 1965, Selected Tables of Atomic Spectra (Washington: NSRDSNBS)

. 1967, Selected Tables of Atomic Spectra (Washington: NSRDS-NBS) NBS) . 1972, A Multiplet Table of Astrophysical Interest (Washington: NSRDS-

. 1993, Tables of Spectra of Hydrogen, Carbon, Nitrogen, and Oxygen Atoms and Ions, ed. J. W. Gallagher (Boca Raton: CRC Press)

Musielok, J., Pawelec, E., Griesmann, U., \& Wiese, W. L. 1999, Phys. Rev. A, 60, 947

Nave, G., Johansson, S., Learner, R. C. M., Thorne, A. P., \& Brault, J. W. 1994, ApJS, 94, 221

Pandey, G. 2006, ApJ, 648, L143

Pandey, G., Lambert, D. L., Jeffery, C. S., \& Rao, N. K. 2006, ApJ, 638, 454 Pandey, G., Lambert, D. L., Rao, N. K., Gustafsson, B., Ryde, N., \& Yong, D. 2004a, MNRAS, 353, 143

Pandey, G., Lambert, D. L., Rao, N. K., \& Jeffery, C. S. 2004b, ApJ, 602, L113

Pandey, G., Rao, N. K., Lambert, D. L., Jeffery, C. S., \& Asplund, M. 2001, MNRAS, 324, 937

Pandey, G., \& Reddy, B. E. 2006, MNRAS, 369, 1677 
Rao, N. K. 2005, in ASP Conf. Ser. 336, Cosmic Abundances as Recods of Stellar Evolution and Nucleosynthesis, ed. T. G. Barnes III \& F. N. Bash (San Francisco: ASP), 185

Rao, N. K., \& Lambert, D. L. 1996, in ASP Conf. Ser. 96, Hydrogen Deficient Stars, ed. C. S. Jeffery \& U. Heber (San Francisco: ASP), 43 2000, MNRAS, 313, L33 2003, PASP, 115,1304

Rao, N. K., Lambert, D. L., \& Shetrone, M. D. 2006, MNRAS, 370, 941

Rao, N. K., \& Lambert, D. L. 2007, MNRAS, in press (arXiv: 0710.5030)

Rao, N. K., Sriram, S., Jayakumar, K., \& Gabriel, F. 2005, J. Astrophys. Astron., 26, 331

Rao, N. K., et al. 2004, Asian J. Phys., 13, 267
Rauch, T., Reiff, E., Werner, K., Herwig, F., Koesterke, L., \& Kruk, J. W. 2006, in ASP Conf. Ser. 348, Astrophysics in the Far Ultraviolet, ed. G. Sonneborn, H. Moos, \& B.-G. Andersson (San Francisco: ASP), 194

Renda, A., et al. 2004, MNRAS, 354, 575

Saio, H., \& Jeffery, C. S. 2002, MNRAS, 333, 121

Tenenbaum, E. D., et al. 2005, AJ, 130, 256

Tull, R. G., MacQueen, P. J., Sneden, C., \& Lambert, D. L. 1995, PASP, 107 , 251

Werner, K., \& Herwig, F. 2006, PASP, 118, 183

Werner, K., Rauch, T., \& Kruk, J. W. 2005, A\&A, 433, 641

Zaniewski, A., Clayton, G. C., Welch, D. L., Gordon, K. D., Minniti, D., \& Cook, K. H. 2005, AJ, 130, 2293 